\section{Serogroup W135 Meningococcal Disease, The Gambia, 2012}

\author{
M. Jahangir Hossain, Anna Roca, \\ Grant A. Mackenzie, Momodou Jasseh, \\ Mohammad Ilias Hossain, Shah Muhammad, \\ Manjang Ahmed, Osuorah Donatus Chidiebere, \\ Ndiaye Malick, S.M. Bilquees, \\ Usman N. Ikumapayi, Baba Jeng, Baba Njie, \\ Mamady Cham, Beate Kampmann, \\ Tumani Corrah, Stephen Howie, \\ and Umberto D'Alessandro
}

In 2012, an outbreak of Neisseria meningitidis serogroup W135 occurred in The Gambia. The attack rate was highest among young children. The associated risk factors were male sex, contact with meningitis patients, and difficult breathing. Enhanced surveillance facilitates early epidemic detection, and multiserogroup conjugate vaccine could reduce meningococcal epidemics in The Gambia.

$\mathrm{M}$ eningococcal disease is endemic to the African "meningitis belt"; outbreaks occur regularly $(1,2)$. Neisseria meningitidis serogroup A causes most (80\%) cases. However, during 2002-2003, serogroup W135 caused a major epidemic in Burkina Faso (attack rate [AR] 251 cases/100,000 population) (3). Thereafter, the incidence of serogroup W135 was low, with isolated cases and a smallscale outbreak in the meningitis belt $(4,5)$. In 2010 , serogroup A conjugate vaccine was introduced into the African meningitis belt and substantially reduced the incidence of meningitis $(6)$.

In The Gambia, only 6 serogroup W135 cases were identified during 1990-1995; the most recent case had been reported in 1995 (7). In 2012, a large epidemic of serogroup W135 occurred throughout the meningitis belt, including The Gambia (1). Most risk factors identified in the meningitis belt concern serogroup A $(8,9)$, and risk factors for serogroup W135 are little studied. Therefore,

Author affiliations: Medical Research Council Unit, Banjul, The Gambia (M.J. Hossain, A. Roca, G.A. Mackenzie, M. Jasseh, M.I. Hossain, S. Muhammad, M. Ahmed, O.D. Chidiebere, N. Malick, S.M. Bilquees, U.N. Ikumapayi, B. Kampmann, T. Corrah, S. Howie, U. D'Alessandro); Ministry of Health, Banjul (B. Jeng, B. Njie, M. Cham); and Institute of Tropical Medicine, Antwerp, Belgium (U. D’Alessandro)

DOI: http://dx.doi.org/10.3201/eid1909.130077 we report the investigation of this epidemic and the related risk factors.

\section{The Study}

The Gambian Ministry of Health and the Medical Research Council Unit, The Gambia, investigated a serogroup W135 epidemic that occurred during February-June 2012 in the Central River Region (CRR) and Upper River Region (URR). Since 2008, surveillance of invasive bacterial diseases has been ongoing in Bansang Hospital in CRR and Basse Health Centre in URR (10). The peripheral health centers refer severely ill patients to these health facilities. Three approaches were used to recruit persons with suspected cases of serogroup W135: enhanced prospective surveillance in Bansang Hospital and Basse Health Centre, retrospective case identification from hospital records, and visits to households with confirmed case-patients serogroup W135 to identify other suspected cases. A suspected case was defined as a history of acute onset of fever and any of the following: altered consciousness, inability to eat, neck stiffness, seizures, petechial rash, or bulging anterior fontanel in a child $<2$ years of age. Cerebrospinal fluid (CSF) and/or blood samples were collected from hospitalized persons with suspected serogroup W135. A confirmed case was a suspected case in which serogroup W135 was identified by culture and/or an antigen-specific test. The alert threshold was defined as $\geq 5$ meningitis cases per 100,000 persons per week; the epidemic threshold was $\geq 10$ cases $(11)$.

The investigation team administered 1 dose of ciprofloxacin to each close contact of confirmed case-patients and provided health information to raise awareness. At the end of the epidemic, The Gambian government deployed the tetravalent meningococcal polysaccharide vaccine.

$\mathrm{CSF}$ and blood samples were cultured for bacteria in BACTEC Medium (Becton Dickinson, Franklin Lakes, NJ, USA) and tested for serogrouping by latex agglutination by using BACTEC and Ramel (Thermo Fisher Scientific, Waltham, MA, USA) test kits. Antimicrobial drug susceptibility was tested.

We conducted a matched case-control (ratio 1:1) study to identify risk factors. Healthy controls were matched by age and village with confirmed case-patients, including those who died. Demographic, socioeconomic, and exposure (within 14 days before illness onset) data were collected by using a structured questionnaire. Risk factors were analyzed by conducting bivariate matched and multivariate conditional logistic regression analyses. The Joint Gambia Government/Medical Research Council Ethics Committee approved the study. All study participants or legal guardians provided written informed consent.

During February 1-June 25, 2012, a total of 469 suspected cases were identified, and 114 were confirmed to be 
Table 1. Confirmed and suspected cases of Neisseria meningitidis serogroup W135, CRR and URR, The Gambia, February 1-June 25, 2012*

\begin{tabular}{|c|c|c|c|c|}
\hline $\begin{array}{l}\text { Health region/case- } \\
\text { patient age group, y }\end{array}$ & Cases, no. (\%) & Deaths, no. (\%) & 2011 population $†$ & Cases/100,000 population \\
\hline \multicolumn{5}{|l|}{ CRR } \\
\hline$<1$ & $62(20)$ & $8(13)$ & 4,216 & 1,470 \\
\hline $1-4$ & $138(45)$ & $4(3)$ & 29,470 & 468 \\
\hline $5-14$ & $70(23)$ & $2(3)$ & 66,545 & 105 \\
\hline$\geq 15$ & 37 (12) & $4(11)$ & 115,995 & 32 \\
\hline $\bar{T}$ Total & $307(100)$ & $18(6)$ & 216,227 & 142 \\
\hline \multicolumn{5}{|l|}{ URR } \\
\hline$<1$ & $47(29)$ & $10(21)$ & 4,086 & 1,150 \\
\hline $1-4$ & $70(43)$ & $5(7)$ & 27,564 & 254 \\
\hline $5-14$ & $36(22)$ & $2(6)$ & 64,014 & 56 \\
\hline$\geq 15$ & $9(6)$ & $1(11)$ & 111,663 & 8 \\
\hline$\overline{\text { Total }}$ & $162(100)$ & $18(11)$ & 207,327 & 78 \\
\hline \multicolumn{5}{|l|}{ CRR and URR } \\
\hline$<1$ & $109(23)$ & 18 & 8,302 & 1,312 \\
\hline $1-4$ & $208(44)$ & 9 & 57,034 & 364 \\
\hline $5-14$ & $106(23)$ & $4(4)$ & 130,560 & 81 \\
\hline$\geq 15$ & $46(10)$ & $5(11)$ & 227,658 & 20 \\
\hline$\overline{\text { Total }}$ & $469(100)$ & $36(8)$ & 423,554 & 111 \\
\hline
\end{tabular}

serogroup W135. Thirty-one were co-primary or secondary cases in confirmed case-patients' households. Most (67\%) suspected case-patients were $<5$ years of age, and $56 \%$ of cases occurred in male patients. The overall case-fatality rate was $8 \%$.

The overall AR was 111 cases per 100,000 persons but was much higher among younger children (Table 1). The epidemic threshold was exceeded in the last week of February and continued until April for persons of all ages and until June for children $<5$ years of age (Figure 1, panels A, B). Among children $<5$ years of age, the peak AR attained 83 and 47 cases per 100,000 persons per week in CRR and URR, respectively (Figure 1, panel B). The epidemic peaked during the high temperature/driest months (March-May) and ended abruptly after the first rainfalls in June (Figure 2).

The most common signs and symptoms among the 113 confirmed serogroup W135 case-patients were weakness $(96 \%)$, irritability $(88 \%)$, neck stiffness $(81 \%)$, and inability to eat $(80 \%)$. Bulging fontanelle $(74 \%)$, altered mental status $(73 \%)$, and seizures $(65 \%)$ occurred in a slightly lower proportion of case-patients.

Blood and/or CSF samples were collected from 301 $(69 \%)$ of 438 hospitalized suspected case-patients, of which almost half (138) were positive for bacterial pathogens. Serogroup W135 was the major pathogen (114 [83\%] of 138); followed by Streptococcus pneumoniae (13\%) and Staphylococcus aureus (2\%). Common antibacterial drugs used for meningitis were tested on $92(81 \%)$ serogroup W135 isolates, which were susceptible to most of them (ampicillin, 100\%; chloramphenicol, 99\%; ciprofloxacin, 99\%; penicillin, $95 \%$; and tetracycline, $92 \%$ ) but not to erythromycin (59\% susceptible) and trimethoprim/sulfamethoxazole ( $100 \%$ resistant).

We enrolled 106 confirmed case-control pairs. Risk factors identified in the univariate analysis were male sex, students, $>4$ children 1-5 years of age in the household, contact with a meningitis case-patient, preceding history of respiratory illness (nasal discharge, difficult breathing), and itchy eyes (Table 2, Appendix, wwwnc.cdc.gov/EID/ article/19/9/13-0077-T1.htm). In the multivariate analysis, male sex (odds ratio [OR] 1.9; 95\% CI 1.0-3.7), contact with meningitis case-patients (OR 4.8; 95\% CI 1.3-17.8), difficult breathing (OR 6.8; 95\% CI 1.4-33.4), and itchy eyes (OR 4.4; 95\% CI 1.3-14.4) remained significantly associated with cases.

\section{Conclusions}

Before the current cases, the most recent sporadic cases in The Gambia were reported in the early 1990s. These cases were part of a larger epidemic in the meningitis belt with a comparable predominance of serogroup W135 followed by S. pneumoniae (1). After introduction of MenAfriVac (serogroup A conjugate vaccine), incidence and epidemics caused by serogroup A decreased substantially in the meningitis belt $(6,12)$. The reemergence of epidemic serogroup W135 in this region requires a strategy for surveillance, epidemic detection and control, and revised vaccination policy.

Serogroup A outbreaks usually affect children $>5$ years of age and young adults $(5,13,14)$. However, two thirds of the serogroup W135 cases occurred in children $<5$ years of age, for whom the AR was 5-fold higher than it was for older age groups, similar to the characteristics of 


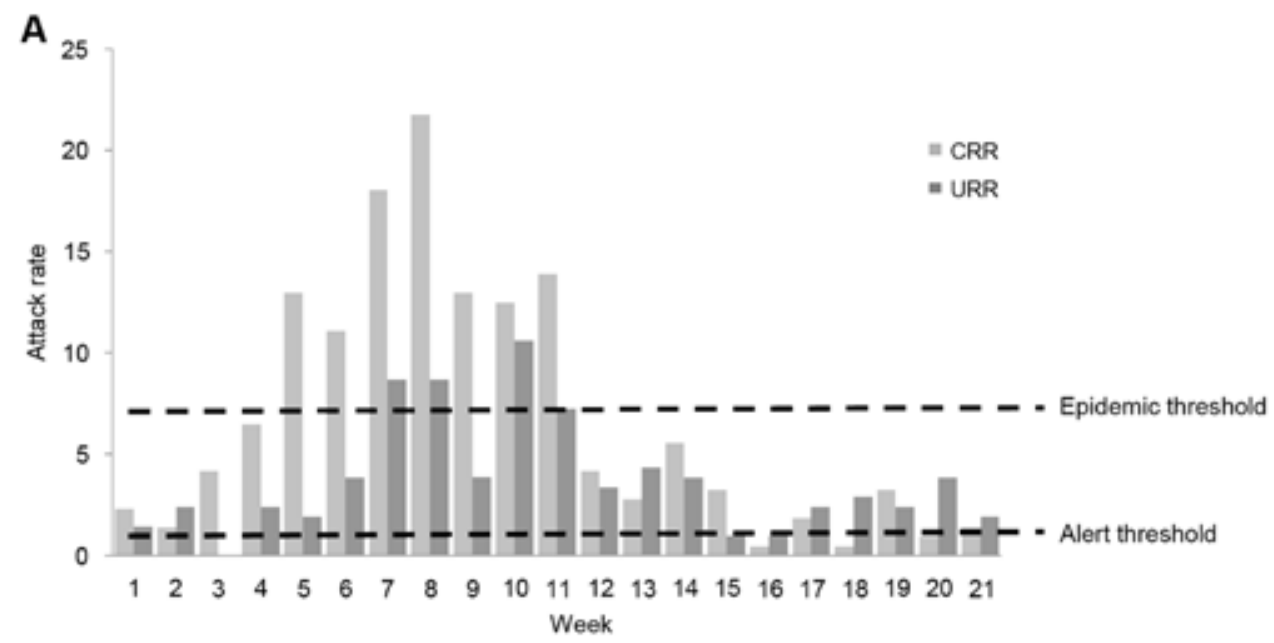

Figure 1. Outbreak of Neisseria meningitidis serogroup W135, Central River and Upper River Regions, The Gambia, February 1-June 25, 2012. A) Attack rate per 100,000 persons per week. B) Attack rate per 100,000 children $<5$ years of age per week. Light gray bars, Central River Region; dark gray bars, Upper River Region. Alert threshold corresponds to an attack rate of 5 ; epidemic threshhold, to an attack rate of 10 .

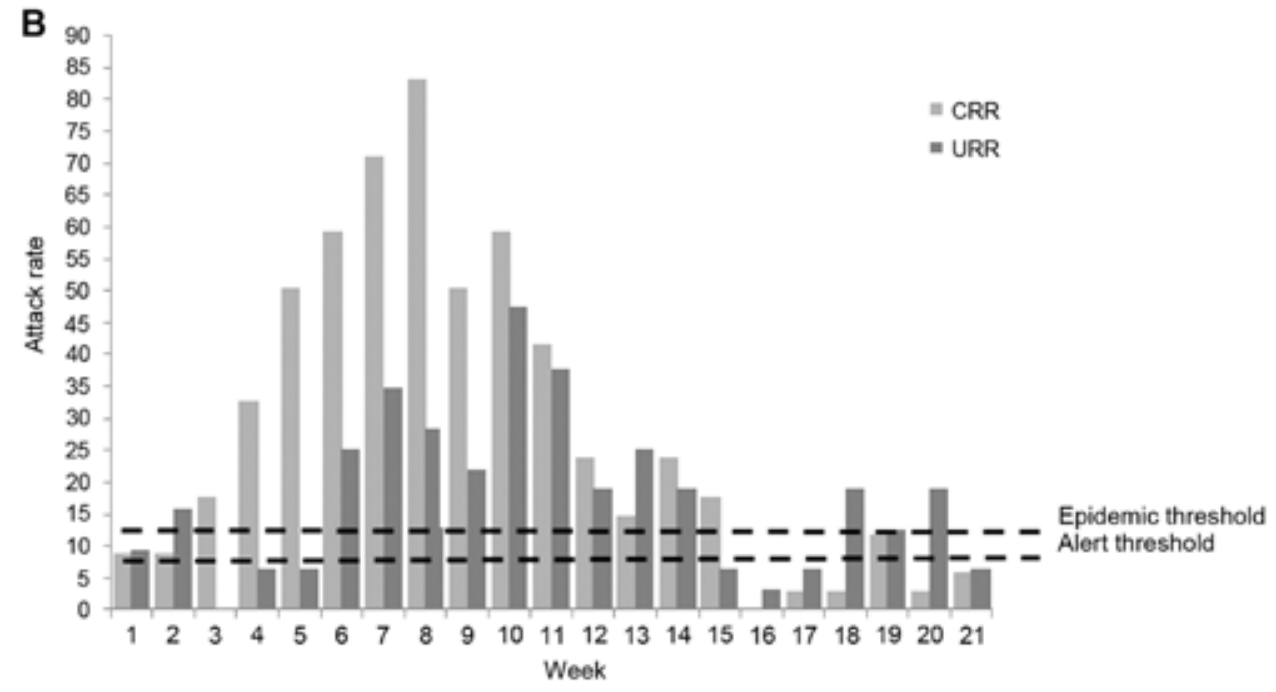

the serogroups W135 outbreaks in Burkina Faso (2002$2003)$ and Niger $(2010)(3,5)$. Therefore, the current operational definition of alert and epidemic thresholds, drawn mostly from serogroup A data, should be revised because merging the AR for all age groups may delay, or result in nondetection of, a serogroup W135 epidemic in younger age groups.

Signs and symptoms of concurrent respiratory illness were more prevalent among case-patients than controls; itchy eyes and difficult breathing were associated with disease. The temporal sequence of these signs relative to the occurrence of meningococcal disease was not determined, and whether these factors facilitated the invasion of serogroup W135 carried in the nasopharynx or whether these symptoms were part of the initial serogroup W135 infection before onset of severe disease is unclear. Contact with confirmed serogroup W135 case-patients was a strong risk factor. These results are consistent with information available for the other serogroups and with the route of serogroup W135 transmission through droplet infection (15).

Our findings suggest that isolation of case-patients and prophylactic treatment of contacts may reduce transmission of meningococcal disease during epidemics. Enhanced surveillance for meningitis is recommended for early detection of epidemics. The occurrence of this large serogroup W135 outbreak suggests that multiserogroup conjugate vaccine should be deployed for control and prevention.

\section{Acknowledgment}

We thank the communities of the CRR and URR of The Gambia, the staff of the government health facilities, the Regional Health Teams, and the Ministry of Health for participating and supporting outbreak investigation. We also thank Lady Chilel Sanyang and Jarrah Manneh for laboratory testing; Golam Sarwar and Sarra Baldeh for organizing the data; and Edrissa Sabally and Yerro Bah for coordinating the field team and data collection. We thank Pa Cheboh Saine for the logistical support. 


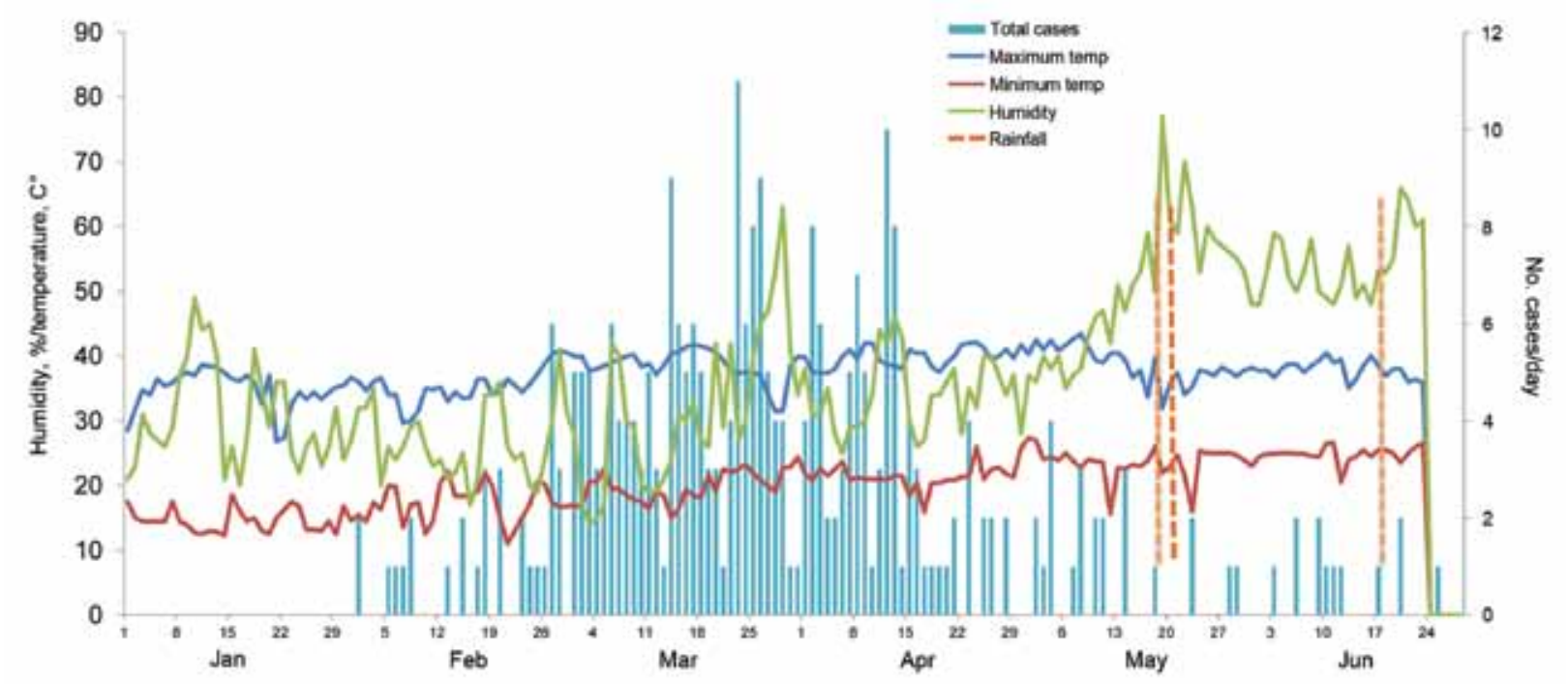

Figure 2. Number of epidemic cases of Neisseria meningitidis serogroup W135 in relation to time, temperature, humidity, and rainfall, Central River Region, The Gambia, February 1-June 25, 2012. A color version of this figure is available online (wwwnc.cdc.gov/EID/article/19/09/130077-F2.htm).

The study was supported by the Medical Research Council. The Bill \& Melinda Gates Foundation funded the prospective bacterial disease surveillance.

Dr Hossain is a clinical epidemiologist and medical graduate working in the Child Survival Theme of the Medical Research Council Unit, The Gambia. His research interests are diarrheal diseases, emerging infections, and infectious diseases.

\section{References}

1. Meningococcal disease in countries of the African meningitis belt, 2012 - emerging needs and future perspectives. Wkly Epidemiol Rec. 2013;88:129-36.

2. Greenwood B. Manson lecture. Meningococcal meningitis in Africa. Trans R Soc Trop Med Hyg. 1999;93:341-53. http://dx.doi. org/10.1016/S0035-9203(99)90106-2

3. Nathan N, Rose AM, Legros D, Tiendrebeogo SR, Bachy C, Bjorlow E, et al. Meningitis serogroup W135 outbreak, Burkina Faso, 2002. Emerg Infect Dis. 2007;13:920-3. http://dx.doi. org/10.3201/eid1306.060940

4. Enhanced surveillance of epidemic meningococcal meningitisinAfrica: a three-year experience. Wkly Epidemiol Rec. 2005;80:313-20.

5. Collard JM, Maman Z, Yacouba H, Djibo S, Nicolas P, Jusot JF, et al. Increase in Neisseria meningitidis serogroup W135, Niger, 2010. Emerg Infect Dis. 2010;16:1496-8. http://dx.doi.org/10.3201/ eid1609.100510

6. Novak RT, Kambou JL, Diomande FV, Tarbangdo TF, OuedraogoTraore R, Sangare L, et al. Serogroup A meningococcal conjugate vaccination in Burkina Faso: analysis of national surveillance data. Lancet Infect Dis. 2012;12:757-64. http://dx.doi.org/10.1016/ S1473-3099(12)70168-8

7. Kwara A, Adegbola RA, Corrah PT, Weber M, Achtman M, Morelli G, et al. Meningitis caused by a serogroup W135 clone of the ET-37 complex of Neisseria meningitidis in West Africa. Trop Med Int Health. 1998;3:742-6. http://dx.doi.org/10.1046/j.13653156.1998.00300.x
8. Hodgson A, Smith T, Gagneux S, Adjuik M, Pluschke G, Mensah $\mathrm{NK}$, et al. Risk factors for meningococcal meningitis in northern Ghana. Trans R Soc Trop Med Hyg. 2001;95:477-80. http://dx.doi. org/10.1016/S0035-9203(01)90007-0

9. Greenwood BM, Greenwood AM, Bradley AK, Williams K, Hassan-King M, Shenton FC, et al. Factors influencing susceptibility to meningococcal disease during an epidemic in The Gambia, West Africa. J Infect. 1987;14:167-84. http://dx.doi.org/10.1016/S01634453(87)92052-4

10. Mackenzie GA, Plumb ID, Sambou S, Saha D, Uchendu U, Akinsola B, et al. Monitoring the introduction of pneumococcal conjugate vaccines into West Africa: design and implementation of a population-based surveillance system. PLoS Med. 2012;9:e1001161. http://dx.doi.org/10.1371/journal.pmed.1001161

11. World Health Organization Regional Office for Africa. Standard operating procedures for enhanced meningitis surveillance in Africa. Version: August 2009 [cited 2012 Mar 5]. http://www.afro. who.int

12. Kristiansen PA, Diomande F, Ba AK, Sanou I, Ouedraogo AS, Ouedraogo R, et al. Impact of the serogroup A meningococcal conjugate vaccine, MenAfriVac, on carriage and herd immunity. Clin Infect Dis. 2013;56:354-63. http://dx.doi.org/10.1093/ $\mathrm{cid} / \mathrm{cis} 892$

13. Greenwood B. Priorities for research on meningococcal disease and the impact of serogroup A vaccination in the African meningitis belt. Vaccine. 2013;31:1453-7. http://dx.doi.org/10.1016/ j.vaccine.2012.12.035

14. Greenwood BM, Bradley AK, Smith AW, Wall RA. Mortality from meningococcal disease during an epidemic in The Gambia, West Africa. Trans R Soc Trop Med Hyg. 1987;81:536-8. http://dx.doi. org/10.1016/0035-9203(87)90397-X

15. Wilder-Smith A, Barkham TM, Earnest A, Paton NI. Acquisition of W135 meningococcal carriage in Hajj pilgrims and transmission to household contacts: prospective study. BMJ. 2002;325:365-6. http://dx.doi.org/10.1136/bmj.325.7360.365

Address for correspondence: M. Jahangir Hossain, Medical Research Council Unit, PO Box 273, Banjul, The Gambia; email: jhossain@mrc.gm 